\title{
Precision laser spectroscopy experiments on antiprotonic helium
}

\author{
Masaki Hori ${ }^{1, *}$ \\ ${ }^{1}$ Max-Planck-Institut für Quantenoptik, Hans-Kopfermann-Strasse 1, D-85748 Garching, Germany
}

\begin{abstract}
At CERN's Antiproton Decelerator (AD) facility, the Atomic Spectroscopy and Collisions Using Slow Antiprotons (ASACUSA) collaboration is carrying out precise laser spectroscopy experiments on antiprotonic helium $\left(\bar{p} \mathrm{He}^{+} \equiv \bar{p}+\mathrm{He}^{2+}+e^{-}\right)$atoms. By employing buffer-gas cooling techniques in a cryogenic gas target, samples of atoms were cooled to temperature $T=1.5-1.7 \mathrm{~K}$, thereby reducing the Doppler width in the single-photon resonance lines. By comparing the results with three-body quantum electrodynamics calculations, the antiproton-to-electron mass ratio was determined as $M_{\bar{p}} / m_{e}=1836.1526734(15)$. This agreed with the known proton-to-electron mass ratio with a precision of $8 \times 10^{-10}$. Further improvements in the experimental precision are currently being attempted. The high-quality antiproton beam provided by the future Extra Low Energy Antiproton Ring (ELENA) facility should further increase the experimental precision.
\end{abstract}

\section{Introduction}

The metastable antiprotonic helium $\left(\bar{p} \mathrm{He}^{+} \equiv \bar{p}+\mathrm{He}^{2+}+e^{-}\right)$atom is an exotic long-lived system made of a helium nucleus, an electron in the ground state, and an antiproton occupying a Rydberg state of principal and orbital angular momentum quantum numbers $n \sim \ell-1 \sim 38$ [1-3]. The unusual longevity of the atom allows us to measure its transition frequencies by laser spectroscopy. By comparing the values with the results of three-body quantum electrodynamics (QED) calculations, the antiproton-to-electron mass ratio $M_{\bar{p}} / m_{e}$ can in principle be determined with a relative precision of $\sim 10^{-11}$ [4-6]. This corresponds to the best determinations of the proton-to-electron mass ratio $M_{p} / m_{e}$ obtained from Penning trap experiments [7-11], or laser spectroscopy of $\mathrm{HD}^{+}$molecular ions [12-14]. The $\bar{p} \mathrm{He}^{+}$experiments also provide a consistency test of CPT symmetry [15], which may be complementary to the spectroscopy experiments on antihydrogen atoms [16-18]. It provides experimental constraints on any exotic fifth force that may exist at the $\sim 1 \AA$ length scale [19-22].

The atoms can be readily synthesized via the reaction, $\bar{p}+\mathrm{He} \rightarrow \bar{p} \mathrm{He}^{+}+e^{-}$, by allowing an antiproton beam [23] to come to rest in a helium gas target [24-26]. The transition frequencies of $\bar{p} \mathrm{He}^{+}$spanning the infrared to ultraviolet range have been calculated [4-6] to a relative precision of $\sim 10^{-10}$ by evaluating the quantum electrodynamics (QED) corrections up to order $m_{e} \alpha^{7}$ in atomic units. Here $m_{e}$ and $\alpha$ respectively denote the electron mass and the fine structure constant. These calculations used the International Council for

\footnotetext{
*e-mail: Masaki.Hori@mpq.mpg.de
} 

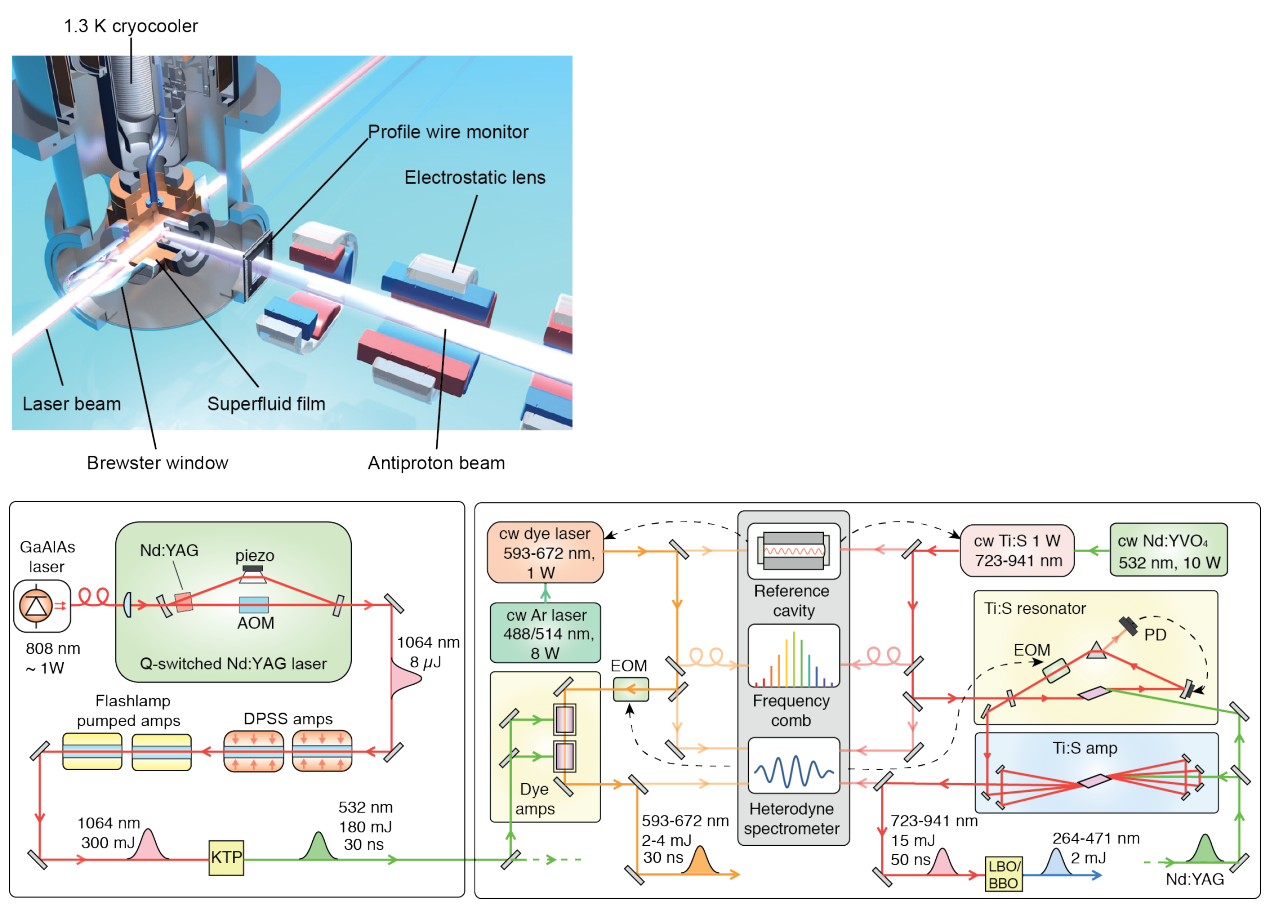

Figure 1. Experimental layout [3] used to synthesize $\bar{p} \mathrm{He}^{+}$and cool them to temperature $T=1.5-1.7$ K (top). Laser system used for single-photon spectroscopy (bottom).

Science Committee on Data for Science and Technology (CODATA) 2010 recommended values of the fundamental constants [27], including the fine structure constant $\alpha$, the ${ }^{3} \mathrm{He}$ - and ${ }^{4} \mathrm{He}$-to-electron mass ratios, the Bohr radius, and the Rydberg constant. The corrections to the transition frequencies that arise from the finite charge radii of the helium nucleus (4 to 7 $\mathrm{MHz})$ and of the antiproton [28] $(<1 \mathrm{MHz})$ are small because the spatial overlap between the Rydberg antiproton orbital and the nucleus is relatively small, and because the antiproton is polarized away from the $1 s$ electron in the atom.

\section{Buffer gas cooling of $\bar{p} \mathrm{He}^{+}$atoms}

The thermal motions of $\bar{p} \mathrm{He}^{+}$in the experimental target at temperature $T$ broadens the width of the measured single-photon laser resonances by a factor, $v \sqrt{8 k_{B} T \log 2 / M c^{2}}$. Here $v$ denotes the transition frequency, $k_{B}$ the Boltzmann constant, $M$ the atom's mass, and $c$ the speed of light. This loss in the spectral resolution limited $[29,30]$ the precision of determining the resonance centroid to around $10^{-7}-10^{-8}$. One way to reach a precision beyond this Doppler limit was provided by two-photon spectroscopy $[2,31]$, in which the $\bar{p} \mathrm{He}^{+}$was irradiated by two counterpropagating ultraviolet laser beams.

An alternative method involved cooling some $2 \times 10^{9} \bar{p} \mathrm{He}^{+}$atoms to a temperature $T=$ $1.5-1.7 \mathrm{~K}$, by allowing the $\bar{p} \mathrm{He}^{+}$to undergo elastic collisions with cryogenic helium gas [3]. This cooling behavior is in contrast to some other kinds of hadronic exotic atoms, such as pionic hydrogen [32], which was found to be heated by collisions with $\mathrm{H}_{2}$ molecules that deexcite the atom. The density of the buffer gas $(T \sim 1.5 \mathrm{~K}$ and $P=40-170 \mathrm{~Pa})$ used in the experiment was carefully adjusted so that the $\bar{p} \mathrm{He}^{+}$atoms, once formed, rapidly underwent a 


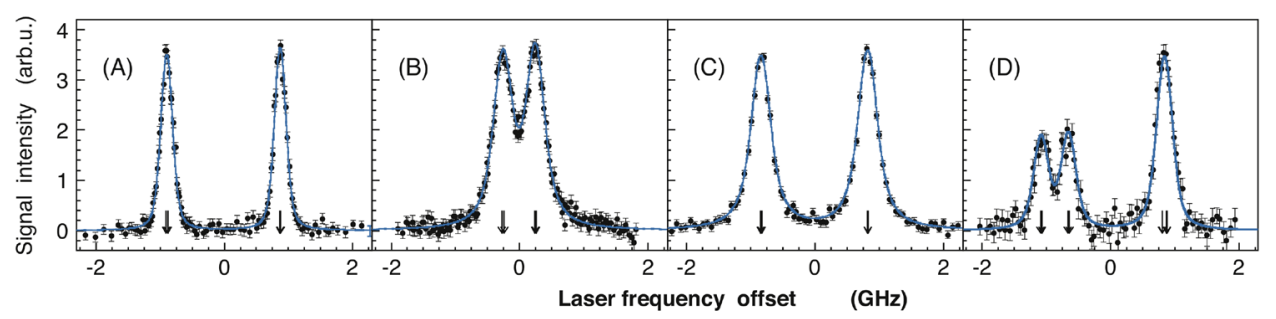

Figure 2. The resonance profiles of the single-photon transitions $(\mathrm{A})(n, \ell)=(37,35) \rightarrow(38,34),(\mathrm{B})$ $(n, \ell)=(39,35) \rightarrow(38,34)$, and $(C)(n, \ell)=(38,35) \rightarrow(39,34)$ of buffer-gas cooled $\bar{p}^{4} \mathrm{He}^{+}$. (D) The resonance profile $(36,34) \rightarrow(37,33)$ of $\bar{p}^{3} \mathrm{He}^{+}$. The $x$-abscissa indicates the offset of the laser frequency relative to the resonance centroid. Solid curves indicate the best fit of an ab initio model based on the optical Bloch equation. From Ref. [3].

few hundred or more cooling collisions. It was then interrogated by the resonant laser beam. The $1 s$ electron protected a significant fraction of the $\bar{p} \mathrm{He}^{+}$from annihilation during this cooling.

The experiment (Fig. 1) was carried out by utilizing the pulsed beam of AD that contained between $2 \times 10^{7}$ and $3 \times 10^{7}$ antiprotons with a kinetic energy $E=5.3 \mathrm{MeV}$ and repetition rate $f=0.01 \mathrm{~Hz}$. Some of the antiprotons were slowed down to $E=75 \mathrm{keV}$ by allowing them to traverse a $3 \mathrm{~m}$ long radiofrequency quadrupole decelerator [29, 33]. This setup was also recently used to attempt to measure the annihilation cross sections of antiprotons that traversed some thin target foils [34-38]. The emerging 75-keV antiprotons were transported by a beamline and allowed to enter the cryogenic helium gas target. The target was in thermal contact with an open-cycle Joule-Thomson cryocooler at temperature $T=1.3 \mathrm{~K}$. The $\bar{p} \mathrm{He}^{+}$ were irradiated by $\Delta t=40$ to $100 \mathrm{~ns}$ long laser pulses [39] with peak powers $P=0.5$ to 10 $\mathrm{kW}$ and wavelengths $\lambda=264$ to $841 \mathrm{~nm}$, which were generated by Ti:Sapphire and dye laser systems.

The spectra in Figs. 2 (A)-(C) show the resonance profiles of the transitions $(n, \ell)=$ $(37,35) \rightarrow(38,34),(39,35) \rightarrow(38,34)$, and $(38,35) \rightarrow(39,34)$ of $\bar{p}^{4} \mathrm{He}^{+}$. They were obtained by plotting the intensities of the antiproton annihilation signals which were induced at multiple laser frequencies between -1 and $1 \mathrm{GHz}$ around the resonance centroid. The arrows indicate the positions of the four hyperfine sublines that arise from the spin-spin interaction between the antiproton and electron in $\bar{p}^{4} \mathrm{He}^{+}$. The single photon resolution seen here exceeds those of sub-Doppler two-photon spectroscopy experiments using higher-temperature atoms [2] described above; this is due to the low $T=1.5-1.7 \mathrm{~K}$ temperature of the atoms. Fig. 2 (D) shows the profile of the $\bar{p}^{3} \mathrm{He}^{+}$resonance $(n, \ell)=(36,34) \rightarrow(37,33)$. The three-peak structure arises from the eight unequally spaced hyperfine sublines caused by the interactions between the ${ }^{3} \mathrm{He}$ nuclear, electron, and antiproton spins. The spin-independent transition frequencies were determined by fitting the profiles with a theoretical line shape (indicated by blue lines). The line shapes were obtained by solving the optical Bloch equations [31] that model the single-photon transition. The experimental uncertainties in the determinations of the $\bar{p} \mathrm{He}^{+}$frequencies include contributions from the statistical uncertainty $( \pm 1 \mathrm{MHz})$ that arises from the limited number of measured atoms, and the systematic uncertainty of 0.4-3 $\mathrm{MHz}$ caused by the fitting function. The dye and Ti:Sapphire lasers used to excite the atoms contain a spurious frequency modulation. These were measured with a precision of 0.4-1.0 MHz. The ac Stark effects induced by the lasers, and magnetic Zeeman shifts due to residual magnetic fields, caused systematic effects of $<0.1 \mathrm{MHz}$ and $<0.2 \mathrm{MHz}$, respectively. 



(B)

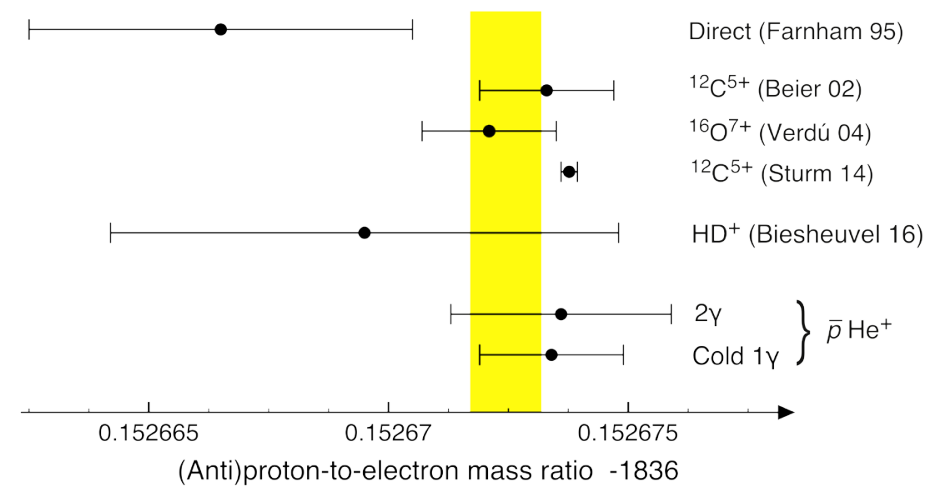

Figure 3. (A) Comparison of experimental and calculated transition frequencies, showing the fractional differences between the experimental (open circles) and theoretical (squares) values of 13 transition frequencies of cooled $\bar{p}^{4} \mathrm{He}^{+}$and $\bar{p}^{3} \mathrm{He}^{+}$atoms. (B) Proton-to-electron mass ratios measured in Penning traps and laser spectroscopy of $\mathrm{HD}^{+}$molecular ions, compared with the antiproton-to-electron mass ratio determined by spectroscopy of $\bar{p} \mathrm{He}^{+}$. The shaded area represents the CODATA 2010 recommended value. From Ref. [3].

Eight transition frequencies of $\bar{p}^{4} \mathrm{He}^{+}$atoms and five frequencies of $\bar{p}^{3} \mathrm{He}^{+}$were measured with relative uncertainties between $2.5 \times 10^{-9}$ and $15 \times 10^{-9}$. The frequencies $v_{\exp }$ (Fig. $3 \mathrm{~A}$, open circles with error bars) agree with theoretical $v_{\text {th }}$ values (filled squares). Due to the cooling techniques described here, this agreement is a factor of 1.4 to 10 times better than previous single-photon experiments [30] of $\bar{p} \mathrm{He}^{+}$. The uncertainties for most of the theoretical frequencies $v_{\text {th }}$ arise from QED contributions of orders higher than $m_{e} \alpha^{7}$ which have not been calculated yet.

When the antiproton-to-electron mass ratio $M_{\bar{p}} / m_{e}$ used in the calculations was changed by $1 \times 10^{-9}$, the theoretical $\bar{p} \mathrm{He}^{+}$frequencies $v_{\text {th }}$ changed by $2.6 \times 10^{-9}$ to $2.7 \times 10^{-9}$. The mass ratio was determined as, $M_{\bar{p}} / m_{e}=1836.1526734(15)$, by minimizing the difference between the frequencies $v_{\text {exp }}$ and $v_{\text {th }}$. The one-standard deviation uncertainty in the parenthesis includes the three contributions $9 \times 10^{-7}, 11 \times 10^{-7}$, and $3 \times 10^{-7}$ of the experimental statistical and systematic uncertainties, and the theoretical uncertainty, respectively.

The atomic mass of the electron was recently determined [10] with a relative precision of $3 \times 10^{-11}$ by confining a ${ }^{12} \mathrm{C}^{5+}$ ion in a Penning trap. The cyclotron frequency of its motion 
in a magnetic field and the precession frequency of the electron spin was then measured, and the results compared with the latest QED calculations of its $g$-factor [40]. From this and the proton mass which was recently measured in a separate measurement involving a Penning trap [11] from the same collaboration, the proton-to-electron mass ratio was determined as, $M_{p} / m_{e}=1836.152673346(81)$. In Fig. 3(B), the latest $M_{p} / m_{e}$ mass ratios are shown together with the previous experimental values that were determined by comparing the cyclotron frequencies of protons and electrons in a Penning trap [7], laser spectroscopy of cold $\mathrm{HD}^{+}$molecular ions [12], and the CODATA 2010 recommended value [27]. These recent high-precision values are in good agreement with the $M_{\bar{p}} / m_{e}$ ratio determined from $\bar{p} \mathrm{He}^{+}$. The TRAP and BASE experiments of CERN have compared the cyclotron frequencies of antiprotons and $\mathrm{H}^{-}$ion pairs confined in a Penning trap [41-43]; a limit of $5 \times 10^{-10}$ was set $[3,44]$ on any deviation between the antiproton and proton masses and charges by combining the results with the $\bar{p} \mathrm{He}^{+}$spectroscopic data.

The $\bar{p}^{4} \mathrm{He}^{+}$transition $(n, \ell)=(40,36) \rightarrow(41,35)$ was also studied by laser spectroscopy [45]. A stimulated first-order Raman scattering process in a $\mathrm{H}_{2}$ gas cell was utilized to generate the $7 \mathrm{~ns}$ long laser pulses of wavelength $\lambda=1154.9 \mathrm{~nm}$ needed to excite this transition. The measurements revealed that most of the metastable populations are concentrated into states of principal quantum number $n \geq 40$, whereas the states $n>41$ contain very few antiprotons. A laser-microwave-laser triple resonance method was used to study the hyperfine structure of the $(n, \ell)=(36,34)$ state of $\bar{p}^{3} \mathrm{He}^{+}[46]$.

\section{Future perspectives}

Currently the precision on the calculations of $\bar{p} \mathrm{He}^{+}$energies are more than an order of magnitude higher than the experimental uncertainties. Further improvements in the theoretical precision are expected in the next 5 years. The Extra Low Energy Antiproton Ring (ELENA) facility due to begin operation in 2021 will provide a high-quality, cooled antiproton beam of energy $E=100 \mathrm{keV}$. Samples of $\bar{p} \mathrm{He}^{+}$would then be formed in a smaller volume, so that lasers of lower power and higher spectral resolution could be used in the experiments. The high stability of the antiproton beam should ensure an improved signal-to-noise ratio on the $\bar{p} \mathrm{He}^{+}$spectral lines. This may allow us to resolve weak atomic transitions between metastable states of smaller natural width, which can be measured with a higher precision. Intensive efforts are currently underway to achieve this goal.

Metastable pionic helium $\left(\pi \mathrm{He}^{+}\right)$is a three-body atom [47-49] consisting of a helium nucleus, an electron in the $1 s$ ground state, and a negative-charged pion in a Rydberg state of quantum numbers $n \sim \ell-1 \sim 16$. The spectral lines of these atoms have never been directly observed, and so the existence of $\pi \mathrm{He}^{+}$is so far hypothetical. Laser spectroscopy of $\pi \mathrm{He}^{+}$is currently being attempted at the $590 \mathrm{MeV}$ ring cyclotron facility of the Paul Scherrer Institute. By comparing the experimental frequencies with those derived from QED calculations, the $\pi^{-}$mass can in principle be determined with a fractional precision of $10^{-8}$ to $10^{-6}$.

\section{References}

[1] R.S. Hayano, M. Hori, D. Horváth, E. Widmann, Rep. Prog. Phys. 70, 1995 (2007)

[2] M. Hori et al., Nature 475, 484 (2011)

[3] M. Hori et al., Science 371, 610 (2016)

[4] V.I. Korobov, L. Hilico, J.-P. Karr, Phys. Rev. Lett. 112, 103003 (2014)

[5] V.I. Korobov, L. Hilico, J.-P. Karr, Phys. Rev. A 89, 032511 (2014)

[6] M-H. Hu et al., Chem. Phys. Lett. 654, 114 (2016) 
[7] D.L. Farnham, R.S. Van Dyck Jr., P.B. Schwinberg, Phys. Rev. Lett. 75, 3598 (1995)

[8] T. Beier et al., Phys. Rev. Lett. 88, 011603 (2002)

[9] J. Verdú et al., Phys. Rev. Lett. 92, 093002 (2004)

[10] S. Sturm et al., Nature, 506, 467-470 (2014)

[11] F. Heiße et al., Phys. Rev. Lett. 119, 033001 (2017)

[12] J. Biesheuvel et al., Nat. Commun. 7, 10385 (2016)

[13] J.C.J. Koelemeij, B. Roth, A. Wicht, I. Ernsting, S. Schiller, Phys. Rev. Lett. 98, 173002 (2007)

[14] S. Alighanbari, M.G. Hansen, V. Korobov, S. Schiller, arXiv:1802.03208 (2018)

[15] V.A. Kostelecký, A.J. Vargas, Phys. Rev. D 92, 056002 (2015)

[16] M. Ahmadi et al., Nature 541, 506 (2017)

[17] M. Ahmadi et al., Nature 548, 66 (2017)

[18] G. Gabrielse et al., Phys. Rev. Lett. 108, 113002 (2012)

[19] E.J. Salumbides, W. Ubachs, V.I. Korobov, J. Mol. Spect. 300, 65 (2014)

[20] J. Murata, S. Tanaka, Class. Quantum Grav. 32, 033001 (2015)

[21] P. Brax, S. Fichet, G. Pignol, arXiv:1710.00850 (2017)

[22] F. Ficek et al., arXiv:1801.00491 (2018)

[23] M. Hori, J. Walz, Prog. Part. Nucl. Phys. 72, 206 (2013)

[24] K. Sakimoto, Phys. Rev. A 91, 042502 (2015)

[25] V.I. Korobov, Z.-X. Zhong, Q.-L. Tian, Phys. Rev. A 92, 052517 (2015)

[26] A. Adamczak, D. Bakalov, Phys. Rev. A 90, 054501 (2014)

[27] P.J. Mohr, B.N. Taylor, D.B. Newell, Rev. Mod. Phys. 84, 1527 (2012)

[28] R. Pohl et al., Nature 466, 213 (2010)

[29] M. Hori et al., Phys. Rev. Lett. 91, 123401 (2003)

[30] M. Hori et al., Phys. Rev. Lett. 96, 243401 (2006)

[31] M. Hori et al., Phys. Rev. A 81, 062508 (2010)

[32] A. Badertscher et al., Phys. Lett. B 392, 278 (1997)

[33] A.M. Lombardi et al., 2001 First operating experience with the CERN decelerating RFQ for antiprotons. Proc. Part. Acc. Conf. Chicago, USA

[34] H. Aghai-Khozani et al., Eur. Phys. J. Plus 127, 125 (2012)

[35] M. Corradini et al., Nucl. Instrum. Methods A 711, 12 (2013)

[36] A. Sótér et al., Rev. Sci. Instrum. 85, 023302 (2014)

[37] K. Todoroki et al., Nucl. Instrum. Methods A 835, 110 (2016)

[38] H. Aghai-Khozani et al., Nucl. Phys. A 970, 366 (2018)

[39] M. Hori, A. Dax, Opt. Lett. 34, 1273 (2009)

[40] K. Pachucki, A. Czarnecki, U.D. Jentschura, V.A. Yerokhin, Phys. Rev. A 72, 022108 (2005)

[41] G. Gabrielse et al., Phys. Rev. Lett. 82, 3198 (1999)

[42] J.K. Thompson, S. Rainville, D.E. Pritchard, Nature 430, 58 (2004)

[43] S. Ulmer et al., Nature 524, 196 (2015)

[44] R.J. Hughes, B.I. Deutch, Phys. Rev. Lett. 69, 578 (1992)

[45] T. Kobayashi et al., J. Phys. B: At. Mol. Opt. Phys. 46, 213 (2013)

[46] S. Friedreich et al., J. Phys. B: At. Mol. Opt. Phys. 46, 125003 (2013)

[47] M. Hori, A. Sótér, V.I. Korobov, Phys. Rev. A 89, 042515 (2014)

[48] V.I. Korobov, A.K. Bakbaev, D.T. Aznabayev, S.A. Zhaugasheva, J. Phys. B: At. Mol. Opt. Phys. 48, 245006 (2015) 
[49] B. Obreshkov, D. Bakalov, Phys. Rev. A 93, 062505 (2016) 